\title{
Advanced structural analysis of a laser additive manufactured Zr-based bulk metallic glass along the build height
}

\author{
James P. Best ${ }^{1,2}$, Keita Nomoto ${ }^{1,3}$, Fan Yang ${ }^{4}$, Bosong $\mathrm{Li}^{1}$, Moritz Stolpe ${ }^{5}$, Luyang Zeng ${ }^{3}$, \\ Zach Evenson ${ }^{6,7,8}$, Christoph Hugenschmidt ${ }^{7}$, Xiaopeng $\mathrm{Li}^{1}$, Simon P. Ringer ${ }^{3}$, and \\ Jamie J. Kruzic ${ }^{1, \star}$ (i) \\ ${ }^{1}$ School of Mechanical and Manufacturing Engineering, University of New South Wales (UNSW Sydney), Sydney, NSW 2052, \\ Australia \\ ${ }^{2}$ Max-Planck-Institut für Eisenforschung GmbH, Max-Planck-Str. 1, 40237 Düsseldorf, Germany \\ ${ }^{3}$ Australian Centre for Microscopy and Microanalysis, and School of Aerospace, Mechanical and Mechatronic Engineering, The \\ University of Sydney, Sydney, NSW 2006, Australia \\ ${ }^{4}$ Institut für Materialphysik Im Weltraum, Deutsches Zentrum für Luft- und Raumfahrt (DLR), 51170 Cologne, Germany \\ ${ }^{5}$ Heraeus Additive Manufacturing GmbH, Heraeusstr. 12-14, 63405 Hanau, Germany \\ ${ }^{6}$ Physik-Department, Technische Universität München, James-Franck-Str. 1, 85748 Garching, Germany \\ ${ }^{7}$ Heinz Maier-Leibniz Zentrum (MLZ), Technische Universität München, Lichtenbergstr. 1, 85748 Garching, Germany \\ ${ }^{8}$ Springer-Verlag GmbH, Tiergartenstr. 17, 69121 Heidelberg, Germany
}

Received: 7 November 2021

Accepted: 8 February 2022

Published online:

4 March 2022

(C) The Author(s) 2022

\begin{abstract}
Additive manufacturing of bulk metallic glasses (BMGs) has opened this material class to an exciting new range of potential applications, as bulk-scale, net-shaped amorphous components can be fabricated in a single step. However, there exists a critical need to understand the structural details of additive manufactured BMGs and how the glassy structure is linked to the mechanical properties. Here, we present a study of structure and property variations along the build height for a laser powder bed fusion (LPBF) processed Zr-based BMG with composition $\mathrm{Zr}_{59.3} \mathrm{Cu}_{28.8} \mathrm{Nb}_{1.5} \mathrm{Al}_{10.4}$ commercially termed AMZ4, using hardness testing, calorimetry, positron annihilation spectroscopy, synchrotron X-ray diffraction, and transmission electron microscopy. A lower hardness, more rejuvenated glassy structure was found at the bottom of the build compared to the middle region of the build, with the structure and properties of the top region between the two. Such differences could not be attributed to variability in chemical composition or crystallisation; rather, the softer bottom region was found to have a larger medium range order cluster size, attributed to
\end{abstract}

Handling Editor: M. Grant Norton.

James P. Best and Keita Nomoto contributed equally to this work

Address correspondence to E-mail: j.kruzic@unsw.edu.au 
heat dissipation into the build plate during processing, which gave faster cooling rates and less reheating compared to the steady-state middle of the build. However, at the top of the build less reheating occurs compared to the middle, leading to a somewhat softer and less relaxed state.

\section{GRAPHICAL ABSTRACT}

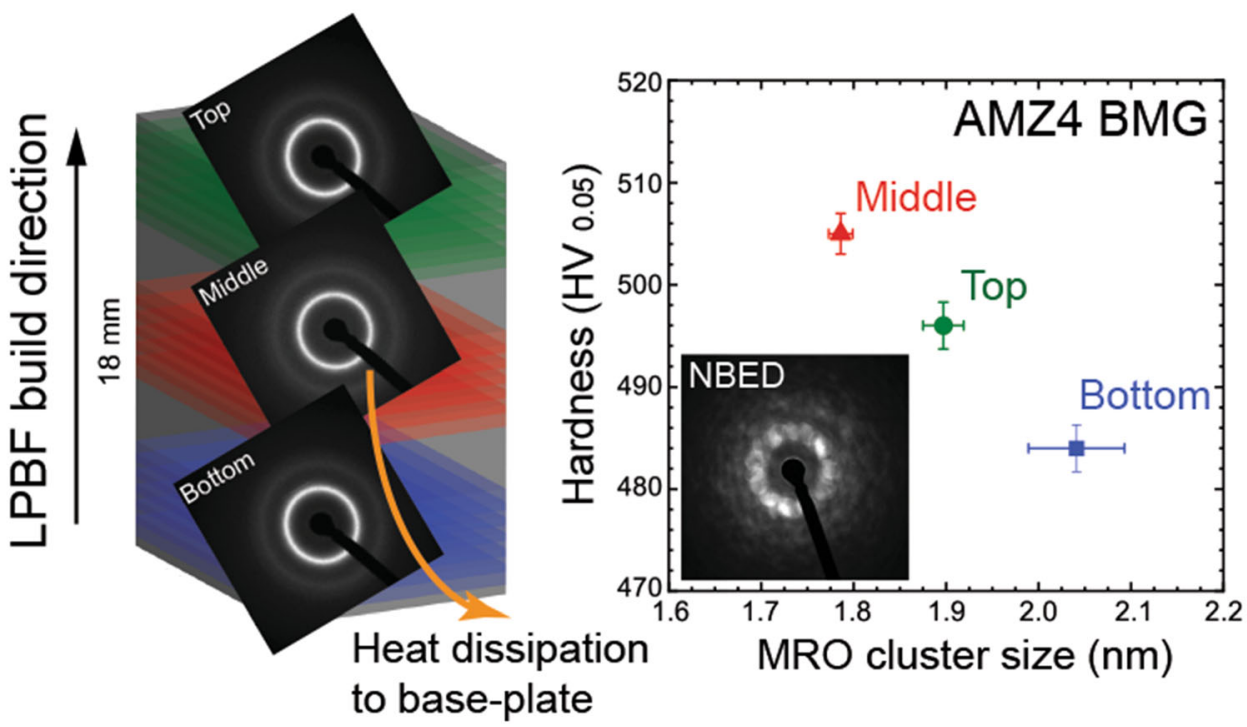

\section{Introduction}

Bulk metallic glasses (BMGs) are alloys with no longrange atomic order but with varying degrees of shortand medium-range order (SRO and MRO). This means that there are no classical lattice defect structures (e.g. grain boundaries or dislocations) giving rise to a fundamental change in the plastic deformation mechanisms, with shear transformations and shear bands dominating rather than dislocation slip and twinning [1]. As such, BMG materials have exceptionally high strengths, elastic strain limits, excellent wear resistance, etc. [2, 3]. A significant bottleneck for the widespread utilisation of BMGs, however, is the rapid cooling rates required during processing to 'freeze-in' the amorphous structure [4]. This requirement has traditionally led to difficulties in producing components with large enough dimensions to be industrially relevant for a range of targeted applications (e.g. for aerospace, transportation, biomedical implants, etc.).

To address this issue, laser powder bed fusion (LPBF) has been recently shown to be a promising method for producing large-scale BMG parts with a broad-range of possible geometries [5-10]. However, there is a growing body of evidence that LPBF-processed parts generally have reduced ductility and damage tolerance when compared with cast alloys of the same chemical composition [10-16]. Since those properties are often seen as limiting factors in the application of BMGs $[2,17]$, such work highlights the importance of understanding the chemical and structural aspects that control the mechanical properties, so that future improvements can be made to LPBF-manufactured BMGs.

The reduced deformation and damage tolerance properties have been attributed in part to various factors such as the presence of crystals, pores, and/or elevated oxygen levels in the bulk amorphous phase [10-16]. However, even for X-ray amorphous samples 
studied with synchrotron micro-diffraction, there is evidence demonstrating microscale heterogeneity in the glassy structure of LPBF-processed BMGs related to the melt-pool solidification process [18]. With regard to improving the fracture toughness of BMGs, the creation of heterogeneous glassy microstructures has proven effective [19-22], and indeed, there is evidence that LPBF-produced BMGs are tougher when cracks are forced to propagate across layer-bylayer heterogeneities [11]. Thus, there is a motivation to better understand the glassy structure variations that exist along the build height of LPBF-produced BMG samples, to enable optimised design and rationalisation of mechanical property variations.

Characterisation studies of LPBF-produced BMGs have mainly focused on layer-level structural variations, such as crystallisation and/or chemical variations in the melt-pool and heat-affected zones (HAZs) or structural periodicity that occurs in the amorphous phase across the build layers [13, 18, 23-25]. In contrast, characterisation studies of traditional crystalline alloys produced by LPBF have often revealed variations in the microstructure at different locations along the build height due to the different heat input and thermal histories that evolve as the build progresses [26-28]. However, little is known about variations that possibly occur along the build height of printed BMG pieces, with one report noting similar porosity levels at the top, middle, and bottom sections of a printed sample [7].

Accordingly, in this work the atomic-scale chemical and structural features were studied at distinct locations (top, middle, and bottom) along the $18 \mathrm{~mm}$ build height of a LPBF-processed BMG alloy (AMZ4- $\mathrm{Zr}_{59.3} \mathrm{Cu}_{28.8} \mathrm{Nb}_{1.5} \mathrm{Al}_{10.4}$ ) using microhardness mapping, differential scanning calorimetry (DSC), Doppler-broadening positron annihilation spectroscopy (DB-PAS), synchrotron X-ray diffraction (sXRD), and transmission electron microscopy (TEM) with nanobeam electron diffraction (NEBD). The combination of these analyses demonstrated that no noticeable structural changes in porosity, chemical composition, and nearest neighbour atomic spacing in the glassy structure were observed along the build height on thin samples cut from the bulk free of residual stresses. However, the bottom of the build had a lower average hardness and wider hardness variability compared to the other locations that was associated structurally with a greater relaxation enthalpy and a larger nanoscale MRO cluster size.
The results of this study improve our understanding of the processing-structure-property relationships for LPBF additive manufactured BMGs.

\section{Material and methods}

\section{Sample fabrication}

A LPBF-processed sample was fabricated using commercially available amorphous AMZ4 powder (10-45 $\mu \mathrm{m}$ particle diameter). The LPBF sample was produced by Heraeus Additive Manufacturing $\mathrm{GmbH}$ (Hanau, Germany) as described in our previous studies [11, 12] using an Electro Optical Systems (EOS) M290 (with a $400 \mathrm{~W}$ Yb-fibre laser) with a classic EOS rotating stripe pattern $\left(67^{\circ}\right.$ scan rotation between each layer) and their proprietary optimised LPBF parameters for a layer thickness of $20 \mu \mathrm{m}$. A specimen with dimensions of $10 \times 10 \times 18 \mathrm{~mm}^{3}$ was fabricated, with the longest dimension corresponding to the build direction. The specimen was welded to a $\sim 25 \mathrm{~mm}$ thick commercially pure Ti base plate using a multiple-pass laser exposure for the first five layers, and no support structures were used. This base plate was screwed into the build-plate holder of the machine. Specimens were cut sufficiently far enough from the plate, at a height of $\sim 0.5 \mathrm{~mm}$, so that the initially welded material should have had no apparent influence on the composition of the final tested material. Subsequent mechanical testing and characterisation was conducted at the top, middle, and bottom of the build where the top and bottom refer to within $4 \mathrm{~mm}$ of the top surface or base plate, respectively, and middle refers to a central $4 \mathrm{~mm}$ along the build direction (Fig. 1).

\section{Mechanical characterisation}

Microhardness mapping was conducted on rectangular cross sections at the top, middle, and bottom of the build that were cut perpendicular to the build direction using a low-speed diamond saw (i.e. indentation surface corresponds to the $x-y$ plane in Fig. 1). A Vickers microhardness indenter was used in an automated hardness tester (Durascan-80, Struers) to create hardness maps with 625 indents each. An applied load of $0.05 \mathrm{~kg}$ and a dwell time of $10 \mathrm{~s}$ were used with an indent spacing of $40 \mu \mathrm{m}$ as described in our previous studies [20, 22, 29]. 


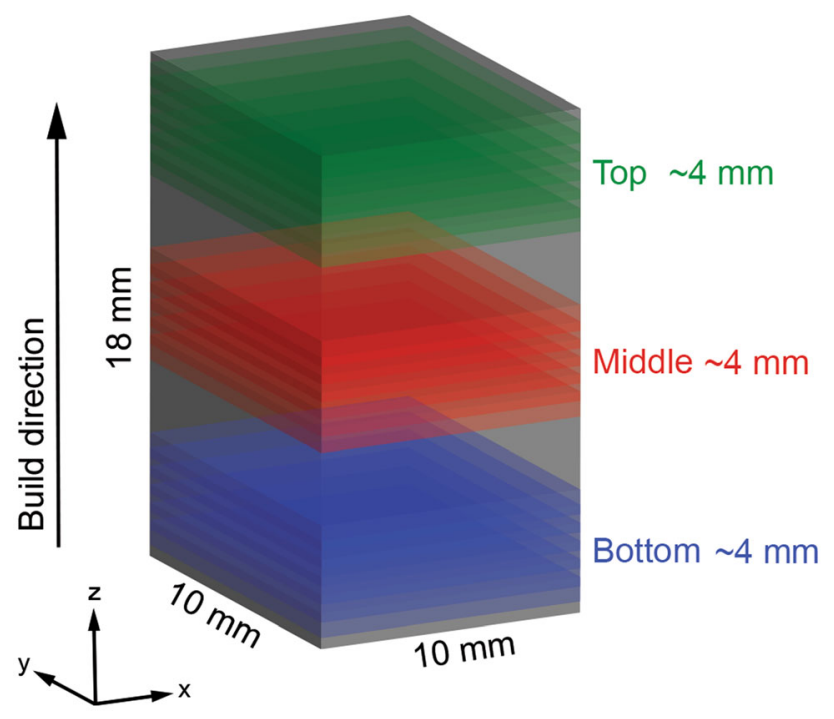

Figure 1 Schematic of the LPBF-processed AMZ4 specimen and the locations of the top, middle, and bottom of the build characterised in this study. With the exception of DB-PAS and SEM/EDS measurements, all specimens were cut perpendicular to the build direction.

\section{Differential scanning calorimetry (DSC)}

Samples for the DSC experiments (DSC 8000, PerkinElmer) with mass of $\sim 20 \mathrm{mg}$ were cut from sections of the built AMZ4 block using a low-speed diamond saw and ground to a 4000 grit finish using $\mathrm{SiC}$ paper to ensure good contact with the bottom of the $\mathrm{Al}$ crucible pan. Samples were cleaned in an ultrasonic cleaner and dried prior to measurement. For each DSC experiment, first the instrument baseline was established as stable and reproducible. Then, the BMG sample was stabilised for $1 \mathrm{~min}$ at $323 \mathrm{~K}$ and then heated from 323 to $863 \mathrm{~K}$ at $20 \mathrm{~K} / \mathrm{min}$. After cooling, the crystalline baseline was obtained for each sample under the identical heating profile and subtracted from the BMG sample scan.

\section{Doppler-broadening positron annihilation spectroscopy (DB-PAS)}

The DB-PAS experiment was performed at the CDBspectrometer [30] at the high intensity positron source NEPOMUC [31] located at the Heinz MaierLeibnitz Zentrum (MLZ) in Garching, Germany. The characteristic S-parameter was obtained using coincident Doppler broadening spectroscopy (CDBS), which is defined as the ratio of counts in a fixed area around the maximum and the total counts of the
$511 \mathrm{keV}$ annihilation line. S-parameters were measured on polished thin $(\sim 200 \mu \mathrm{m})$ cross sections of the samples with a bias voltage set to $-30 \mathrm{kV}$ and a positron beam of approximately $100 \mu \mathrm{m}$ in diameter. Line scans were performed along the build direction, with a step size of $150 \mu \mathrm{m}$.

\section{High-energy synchrotron X-ray diffraction (sXRD)}

Measurements were performed in the second experimental hutch of the PETRA III P07 beamline at the Deutsches Elektronen-Synchrotron (DESY) in Hamburg, Germany. For measurement, rectangular samples with maximal dimensions of approximately $2 \mathrm{~mm}$ (thickness $\sim 200 \mu \mathrm{m}$ ) were cut using a lowspeed saw from the bottom, middle, and top sections of the built block perpendicular to the build direction. Samples were then ground with 4000 grit $\mathrm{SiC}$ paper to remove surface asperities.

Spatially resolved X-ray diffraction line-scans were performed using a micro-focussed monochromatic photon beam with an energy of $98.15 \mathrm{keV}$ and a size of $30 \times 2 \mu \mathrm{m}^{2}(w \times h)$ attained using an $\mathrm{Al}$ compound refractive lens system in transmission mode. The resultant $X$-ray scattering patterns were collected using an exposure time of $4 \mathrm{~s}$ on a PILATUS3 $\mathrm{X}$ CdTe $2 \mathrm{M}$ flat panel detector placed about $400 \mathrm{~mm}$ downstream of the sample. The diffraction patterns showed a fully amorphous structure absent of any intermetallic or oxide crystalline peaks. A $2 \mu \mathrm{m}$ stepsize along the $x$-axis was used perpendicular to the sample build direction $(x=0.2 \mathrm{~mm})$, and a $30 \mu \mathrm{m}$ step-size was used along the $z$-axis $(z=0.09 \mathrm{~mm})$, totalling $100 \times 3$ diffraction images per sample. The collected 2D diffraction patterns were integrated over the full azimuthal range using the Python pyFAI package [32], with a calibration using $\mathrm{CeO}_{2}$ standard. Measured intensity data $I_{\mathrm{m}}(Q)$ as a function of scattering vector $Q$ were then processed using PDFget X3 [33] to allow for the determination of the structure function $S(Q)$. As described in Reference [18], the measurements were corrected for sample absorption, X-ray polarisation, Compton scattering, and any background scattering from the sample container or environment. The peak position of $S(Q)$ was determined by an interpolation using a third-order polynomial in a limited $Q$ range close to the maximum. 


\section{Electron microscopy}

Scanning electron microscopy (SEM) with energydispersive spectroscopy (EDS) was done to further confirm the chemical homogeneity in the melt pools and heat-affected zones (HAZs) found in our previous study [18]. The X-ray energy spectra were collected at $20 \mathrm{kV}$ and $0.8 \mathrm{nA}$ with a dwell time of $100 \mu \mathrm{s}$ using X-Max $80 \mathrm{~mm}^{2}$ EDS detector (Oxford Instrument, UK). Transmission electron microscopy (TEM) specimens from the bottom, middle, and top sections of the built specimen were prepared by two different methods: (1) twin-jet electropolishing (TenuPol-5, Struers, USA) with $10 \%$ nitric acid in methanol at $-20{ }^{\circ} \mathrm{C}$ and $30 \mathrm{~V}$ and (2) a standard lift-out method using a Xenon plasma focused ion beam (pFIB) scanning electron microscope (PFIB-SEM, Helios G4 PFIB, Thermo Fisher Scientific, USA). The pFIB samples were specifically used for site-specific experiments where TEM lamellae were extracted between four indents of nearly identical microhardness as determined by the microhardness mapping procedures described above. TEM analyses were performed using a JEM-2200FS (JEOL, Japan) at an acceleration voltage of $200 \mathrm{kV}$. Experiments for calculating TEM-based PDFs were conducted by selected area electron diffraction (SAED). The SAED patterns were acquired at an exposure time of $2 \mathrm{~s}$ using an energy slit of $5 \mathrm{eV}$ together with a selected area aperture $(10 \mu \mathrm{m})$ and entrance aperture $(120 \mu \mathrm{m})$. The extracted azimuthally integrated diffraction patterns from the SAED patterns were first converted to structure functions, $S(Q)$, and then pair distribution functions (PDFs), $G(r)$, using [34-36]:

$G(r)=\frac{2}{\pi} \int_{Q_{\min }}^{Q_{\max }} Q[S(Q)-1] \sin (Q r) \mathrm{d} Q$,

and an in-house developed software called EDP2PDF, where the camera length was calibrated at $300 \mathrm{~mm}$ in diffraction mode using the (200) plane of gold standard nanoparticles to accurately compare each dataset.

The MRO cluster size was measured by nanobeam electron diffraction (NBED) and fluctuation electron microscopy (FEM) [37-41]. For each measurement, 225 NBED images were converted to mean images and a normalised variance image using [37]:
$V\left(k, Q_{M}\right)=\frac{\left\langle I\left(k, Q_{M}\right)^{2}\right\rangle}{\left\langle I\left(k, Q_{M}\right)\right\rangle^{2}}-1$,

where $I$ is the diffracted intensity from NBED, $V$ the normalized variance, $k$ the scattering vector, $Q_{M}$ the radius of a virtual objective aperture, and $<>$ represents the average over all 225 diffracted images. A nanobeam diameter, $R$, range of $0.7-1.6 \mathrm{~nm}$ was used in this study and $Q_{M}$ is given by $Q_{M}=0.61 / R$ [37]. The annular integrated variance and mean were calculated on a pixel by pixel basis [42] as a function of the $k$-value using PASAD tools [43] and a custom script in DigitalMicrograph [44]. The first two peak positions of $V\left(k, Q_{M}\right)$ were read at approximately $3.6 \mathrm{~nm}^{-1}$ and $4.5 \mathrm{~nm}^{-1}$ using a custom MATLAB script, and the error analysis for the MRO cluster size is explained in our previous study [29]. The pair persistent model was then used to extract MRO cluster/paracrystal diameter in our BMG samples [40]. In this model, the plot of the calculated values of $Q_{M}^{2} / V$ against $Q_{M}^{2}$ shows a linear trend [38]:

$\frac{Q_{M}^{2}}{V\left(k, Q_{M}\right)}=\frac{1}{P(k) \Lambda^{3}}+\frac{4 \pi^{2}}{P(k) \Lambda} Q_{M}^{2}$,

where $\Lambda$ is a characteristic length scale:

$\Lambda=\frac{1}{2 \pi} \sqrt{\frac{m}{c}}$,

that can be extracted from the slope $m$ and intercept $c$ of the line fit to $Q_{M}^{2} / V$ versus $Q_{M}^{2}$. Gibbson et al. [40] suggested that the $\Lambda$ corresponds to the radius of ordered regions. If we assume it as the radius of gyration for spheroidal regions, the width $W$ of the cluster/paracrystal (MRO in this case) can be estimated from the relation.

$W=\sqrt{10} \Lambda$.

Further experimental details can be found in our previous study [29].

\section{Results}

Our previous investigations of a comparable LPBFprocessed AMZ4 sample indicated uniform porosity and chemistry along the build height, though fluctuations in structure and elastic modulus exist layerby-layer corresponding to the melt pool thickness [18]. Analysis of other samples produced by the same LPBF-processing parameters have further confirmed 
the uniformity in chemistry and porosity [11], and no chemical variations were found after doing further EDS in this study (e.g. see Supplementary Materials Fig. S1). Figure $2 \mathrm{a}-\mathrm{c}$ shows the microhardness map for the top, middle, and bottom of the build on the sample cross-sections, respectively. Some indent points were measured as very soft spots (e.g. below $\left.400 \mathrm{HV}_{0.05}\right)$ due to pores produced by the LPBF process and were not included in subsequent averaging. Comparing the overall hardness for the three build positions, the bottom region had the lowest average hardness $\left(491.6 \pm 9.7 \mathrm{HV}_{0.05}\right)$, which can be seen in the light blue and green colour distributed on the map, while the top and middle regions have an average hardness $\mathrm{HV}_{0.05}$ of $494.7 \pm 8.3$ and $497.4 \pm 8.4$, respectively. Figure $2 \mathrm{~d}-\mathrm{f}$ shows Vickers microhardness distribution for each build location. While all three distributions overlap, the bottom of the build contains an enhanced relative frequency of lower hardness points $\left(\sim 470-490 \mathrm{HV}_{0.05}\right)$ and there is a perceptible shift of the hardness distribution to lower values compared to the top and middle of the build.

Representative DSC curves are displayed in Fig. 3. Although two repeat measurements were performed per sample position, the collected data were found to be repeatable and thus single curves are compared for clarity. Differences between all curves were found to be small; however, some variation was observed for the relaxation enthalpy prior to the glass transition temperature, $T_{\mathrm{g}}$, and also in the crystallisation temperature, $T_{x}$. The relaxation enthalpy $(\Delta \phi)$ was determined for each curve by integrating the area between the DSC curve and a horizontal line drawn starting at the heat flow at $230{ }^{\circ} \mathrm{C}$. The DSC data from the bottom region of the build had the highest relaxation enthalpy at approximately $-8.9 \mathrm{~J} / \mathrm{g}$, followed by the top region at approximately $-7.1 \mathrm{~J} / \mathrm{g}$. The middle region showed the lowest relaxation enthalpy at approximately $-6.6 \mathrm{~J} / \mathrm{g}$, along with a slight $T_{\mathrm{x}}$ shift to higher temperatures $\left(\Delta 0.7^{\circ} \mathrm{C}\right)$. The increased relaxation enthalpy magnitude in the lower $\sim 4 \mathrm{~mm}$ of the build suggests a significantly (a) Top

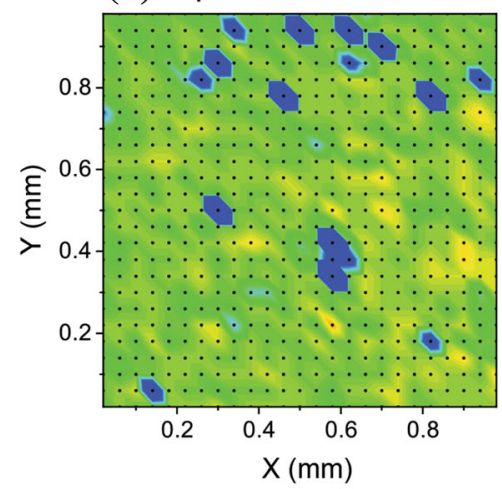

(b) Middle

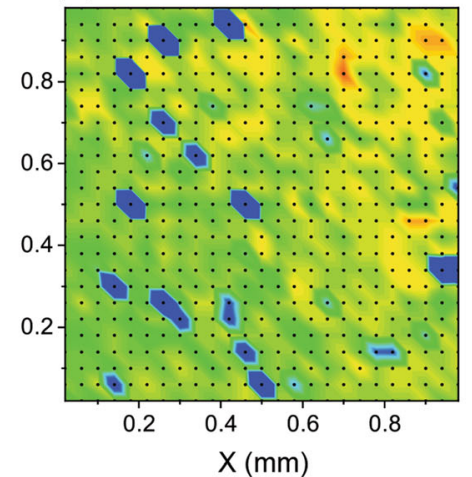

(c) Bottom

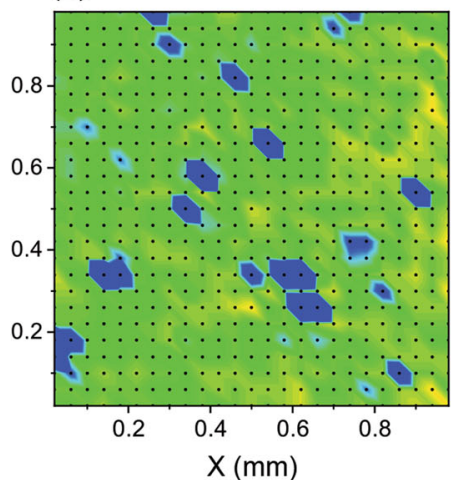

HV 0.05

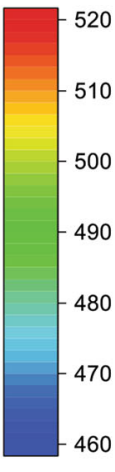

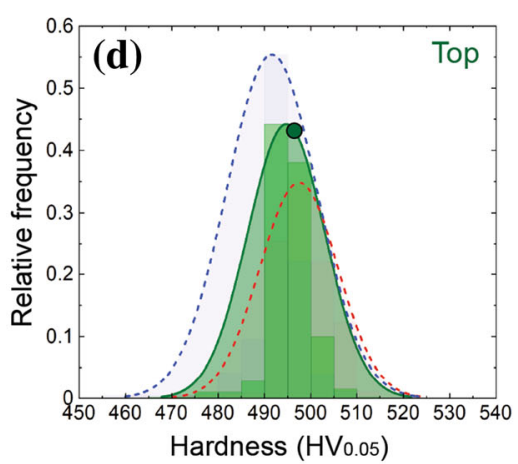
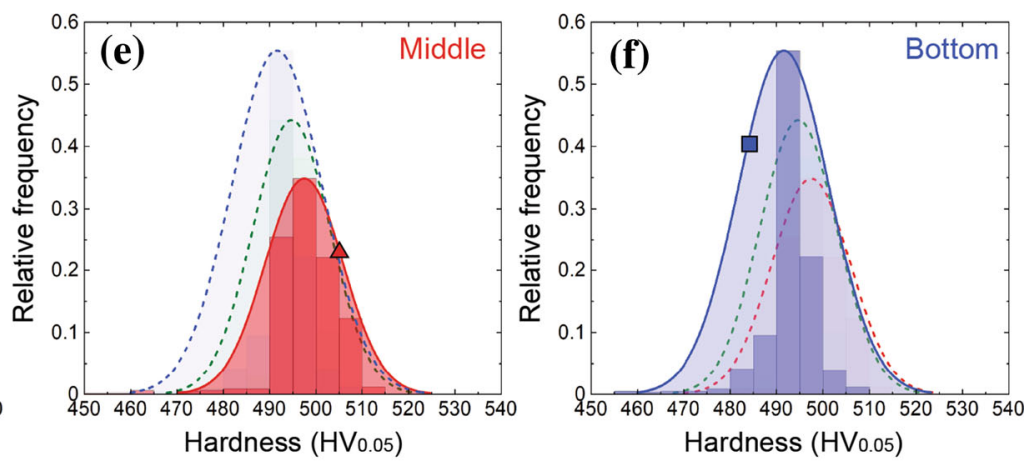

Figure 2 Microhardness maps from the top (a), middle (b), and bottom (c) of the LPBF-processed AMZ4. Approximately 625 indent points were measured for each map, and indents affected by pores (dark blue spots below $400 \mathrm{HV}_{0.05}$ ) were removed from further analysis. (d-f) Vickers microhardness histogram and fitted normal distribution from the top, middle, and bottom of the sample. Symbols in $(\mathbf{d}-\mathbf{f})$ indicate the hardness values where FIB samples were taken for TEM analysis of the MRO. 
Figure 3 DSC traces for samples taken from the top, middle, and bottom of the LPBF AMZ4 build (a). Magnified view of the DSC curves near the glass transition temperature (b) and crystallisation transition (c). Also shown are the relaxation enthalpy $(\Delta \phi)$ values and the crystallisation temperature range $\left(T_{\mathrm{x}}\right)$.

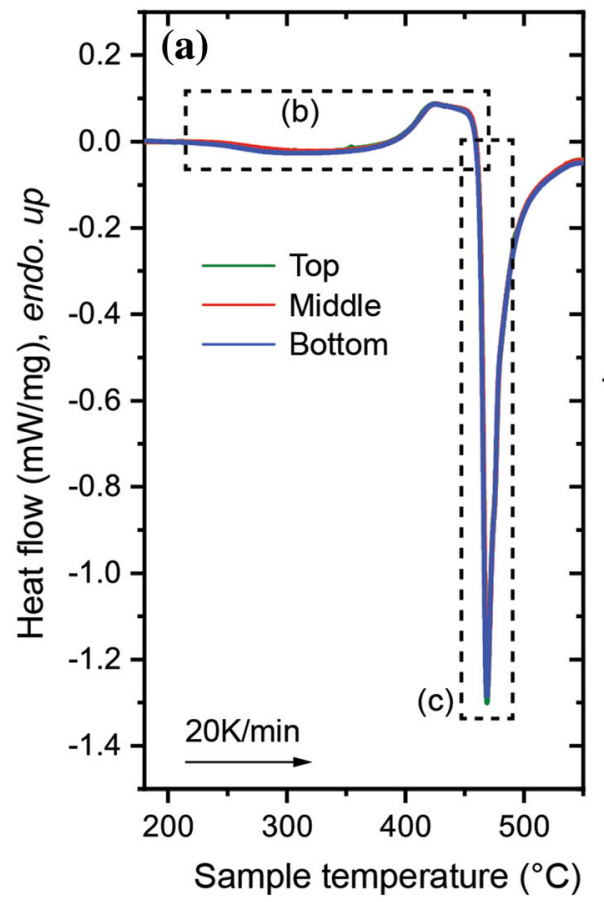

less relaxed structural state with higher levels of free volume that should reduce the flow stress [45]. Indeed, comparisons of the hardness and relaxation enthalpy results in this study confirm the less relaxed state at the bottom of the build is softer.

To investigate this structural difference observed from DSC in increased detail, DB-PAS was used to monitor free volume difference from the bottom of the build in comparison to the middle portion, i.e., where the largest differences are expected. Figure 4 shows the measured S-parameter obtained from DB-PAS along the build direction over a distance of $10 \mathrm{~mm}$, for two parallel line-scans separated by $100 \mu \mathrm{m}$. The S-parameter scatters around a mean value of $\sim 0.578$, where the scattering can be attributed to the layered structure present in the printed sample. However, unlike in our previous sXRD measurements [18], no clear periodicity of the variation related to the layer thickness or HAZ can be recognised, owing to the relatively large size of the positron beam which is on the order of the layer thickness. Nevertheless, for the scanning position in the range corresponding to the bottom part of the build, the mean $S$ value is perceptibly higher than that measured in the middle of the build and is emphasised with a smoothed adjacent-averaged curve as the mean values are within a single deviation. It should be noted that in our previous DB-PAS

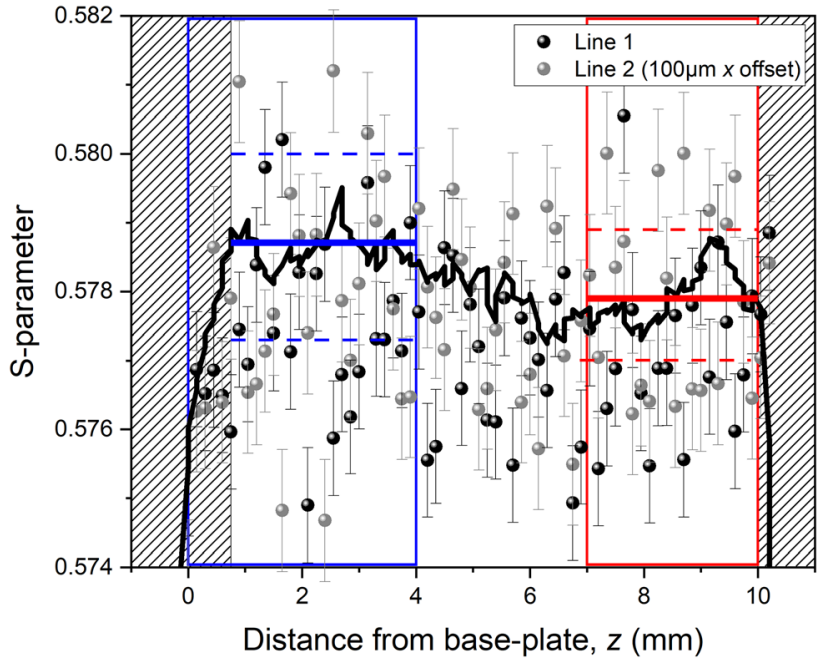

Figure 4 S-parameter obtained from DB-PAS on the LPBFprocessed AMZ4 specimen. Two line-scans are shown over a scan distance of $\sim 10 \mathrm{~mm}$ from the base plate covering the transition from bottom (blue box) to middle (red box) of the specimen. Hashed areas indicate where the measurement was affected by scattering from the Al frame holding the samples. Mean and standard deviations are indicated for both regions, while an 8-pt. adjacent average for both data sets (black line) highlights the subtle transition of the S-parameter from the bottom to middle of the build.

investigation between LPBF and cast AMZ4, the rapidly cooled LPBF material has an S-parameter $0.5 \%$ greater than the cast [12]; differences here along 
build height are expected to be small. However, the slightly higher S-parameter at the bottom of the build indicates a state of more trapped excess free volume, which is in line with that observed in the DSC measurement as well as the lower average hardness value found for that region.

Figure 5 shows the position of the first structure function $S(Q)$ maximum as a function of scan position from three representative sXRD scans for the top, middle and bottom parts of the build. Despite using a scanning direction perpendicular to our previous work, similar periodic variations caused by heterogeneities from the HAZs can be recognised [18]. This was attributed to the 3-dimensional shape of the melt pools and data collection is expected to cross many HAZs for almost any scan direction. The samples exhibited structure factors characteristic of fully amorphous materials. There was negligible average variation in $S(Q)$ peak positions when comparing the various line scans, indicating that significant differences in oxygen content between build locations can be ruled out based on our previously reported observations of $S(Q)$ for AMZ4 samples with varying oxygen content [46]. Furthermore, no significant statistical differences in $S(Q)$ peak positions were observed for the different regions of the build. Averaging all scans for the bottom, middle, and top part of the build (300 data points per region) gives mean positions of the first structure function maximum of $2.597 \pm 0.002 \AA^{-1}, 2.596 \pm 0.004 \AA^{-1}$, and $2.596 \pm 0.002 \AA^{-1}$, respectively, which does not

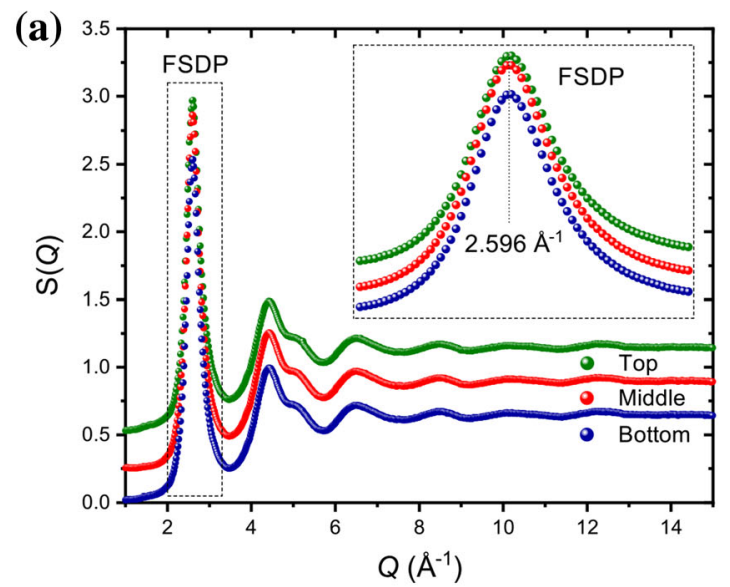

Figure 5 High-energy X-ray micro-diffraction results for the $S(Q)$ scanned on thin cross-sections of material with data collection steps perpendicular to the build direction. Shown are directly correspond to the trends observed in microhardness, DSC, or DB-PAS experiments.

Figure 6 shows representative bright-field (BF) high-resolution TEM (HRTEM) images and selected area electron diffraction (SAED) patterns of the LPBF AMZ4 samples from the top, middle, and bottom of the build. A typical amorphous structure was observed for all of the samples. By searching a large field of view around the edge of the electropolished TEM lamella, no evidence of nanocrystalline phase formation was found at the melt-pools, HAZs, or the matrix. Similarly, searching even larger areas using SEM and EDS further confirmed the melt pools and HAZs were compositionally homogeneous (see Supplementary Materials Fig. S1). While sub-micrometre sized crystals could be found in some HAZs by searching in the SEM, they were isolated and few enough to not appear in the XRD patterns nor in the TEM samples and they did not significantly affect the DSC crystallisation peak magnitudes. Thus, the difference in the hardness response with the build position observed in Fig. 2 was not attributed differences in chemical composition or crystallinity at the micro- or nanoscale.

Figure 7 compares the TEM-based structure functions, $S(Q)$, and PDFs for the top, middle, and bottom of the build. The observed structural changes in the DSC measurement did not result in measurable shifts in the TEM-based $S(Q)$ and PDF nearest neighbour peak positions. While TEM-based PDF analysis has the advantage of collecting local structural information via SAED patterns, the ability of the subsequent

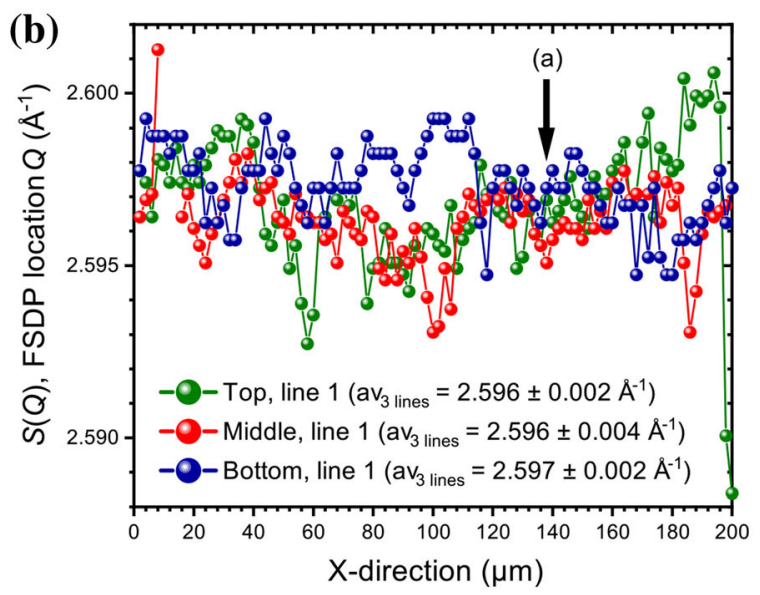

representative $S(Q)$ curves extracted from the diffraction data (a), along with peak positions for the first significant diffraction peak (FSDP) from a line scan over the sample (b). 

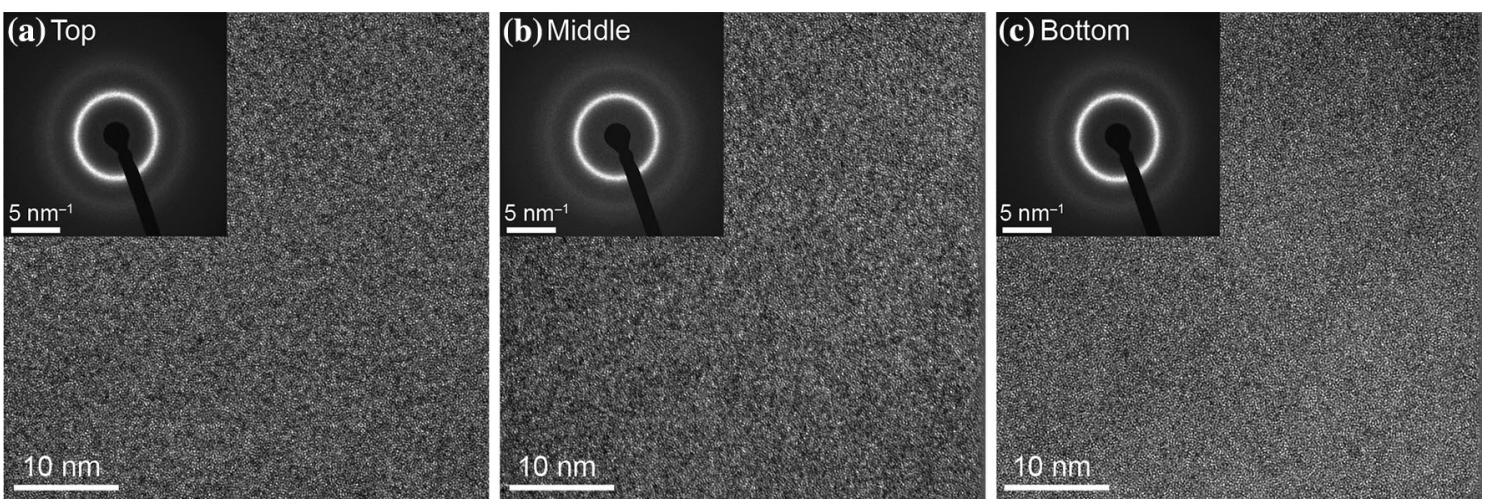

Figure 6 BF-HRTEM images and SAED patterns at different regions of the build: a top, $\mathbf{b}$ middle, and $\mathbf{c}$ bottom.

Figure 7 TEM-based a structure function and $\mathbf{b}$ pair distribution function for the top, middle, and bottom of the build. The 1 st NN represents 1st nearest neighbour interatomic distance.
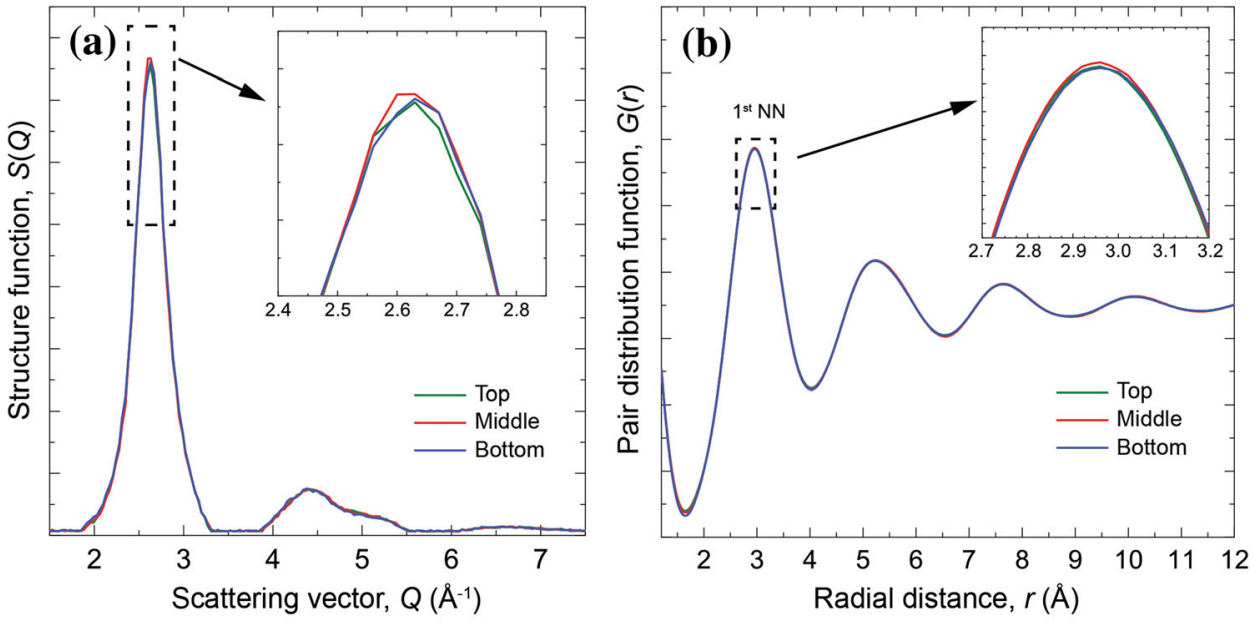

PDF analysis from SAED patterns to detect the fine structure is relatively insensitive compared with that from synchrotron X-ray diffraction analysis [47-49]. Similar to the synchrotron $\mathrm{X}$-ray diffraction results seen in Fig. 5, there were no clear trends of changing TEM-based structure function or nearest neighbour position with the build height that correspond to the observed trends in hardness, relaxation enthalpy, and S-parameter.

The microhardness maps in Fig. 2 and the DSC results in Fig. 3 showed variations in the hardness and structure between the top, middle, and bottom of the build, while the short-range order nearest neighbour distances and local chemistry were identical. To better understand the structure and property relationship, the medium-range order (MRO) cluster size results were examined using NBED experiments and FEM as presented in Fig. 8. Figure 8a shows an example NBED pattern from the top of the build. With more than 200 NBED patterns, the normalised variance patterns were generated and converted into a 1D profile, $V$, as a function of scattering vector, $k$, as shown in Fig. $8 b$. The different nanobeam sizes, $R$, from $0.7 \mathrm{~nm}$ to $1.6 \mathrm{~nm}$ allow us to generate $Q_{M}^{2} /$ $V$ versus $Q_{M}^{2}$ plots $(Q=0.61 / R)$ to extract the $\mathrm{MRO}$ cluster size. Figure $8 \mathrm{c}$ displays the MRO cluster size using electropolished TEM samples from the top, middle, and bottom of the build. The MRO cluster size from the bottom position was larger than that from the top and middle. Note that the hardness values for the electropolished TEM samples were unknown and the NBED patterns were collected from different locations near the edge of the thin area of the samples. In Fig. 8b, the TEM lamellae were taken between four indents of nearly identical hardness to correlate the MRO cluster size and the local hardness. The specific local hardness values for each position were plotted on the curves with circle (top), triangle (middle), and square (bottom) marks in Fig. 2d-f. A clear trend was found of increasing MRO cluster size with decreasing hardness. The linear 

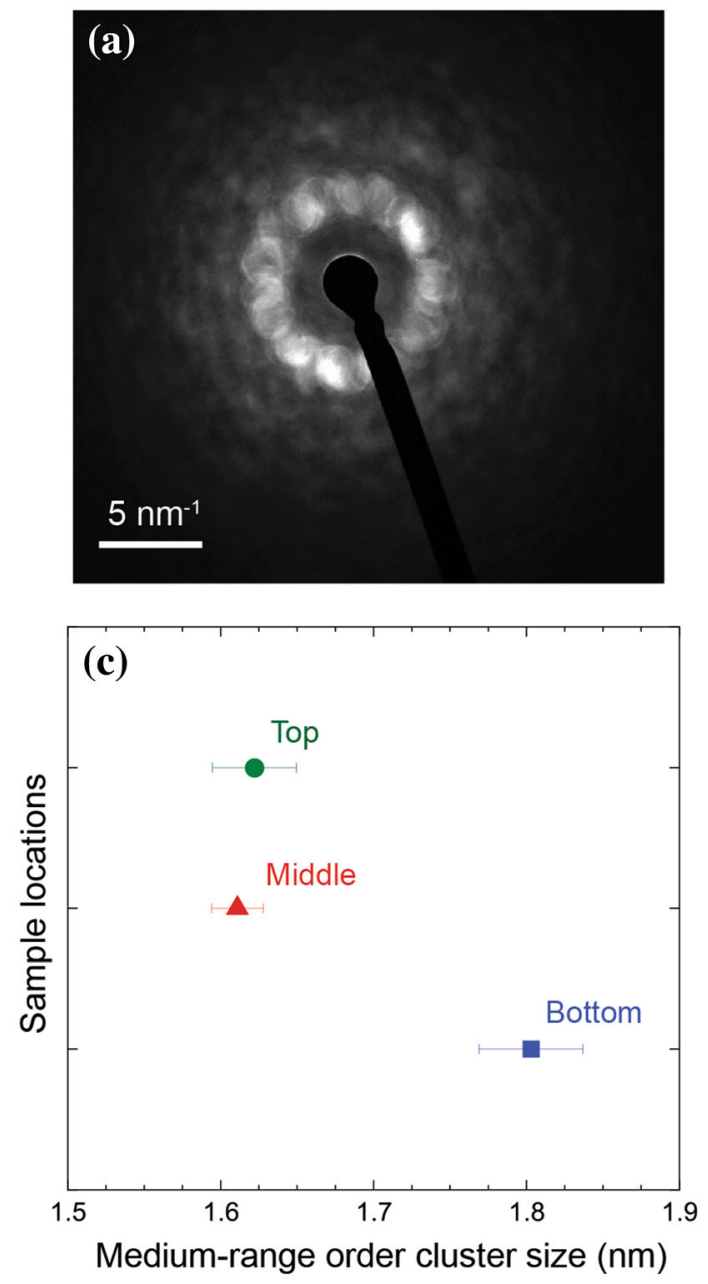

Figure 8 Nanobeam electron diffraction (NBED) and fluctuation electron microscopy (FEM). (a) Example of NBED pattern using a $1.0 \mathrm{~nm}$ electron nanobeam. (b) Normalised variance profiles and $Q_{M}^{2} / V$ versus $Q_{M}^{2}$ plots (inset). The electron beam diameter was varied from $0.7 \mathrm{~nm}$ to $1.6 \mathrm{~nm}$ to obtain the plots and the normalised variance profiles were azimuthally integrated into $1 \mathrm{D}$ profiles. The peak positions of the normalized variance profiles were read at $3.58 \mathrm{~nm}^{-1}$ and $4.60 \mathrm{~nm}^{-1}$. The sample used in (a) and

correlations between the local hardness and MRO cluster size were the same found in Ref. [29].

\section{Discussion}

The present study identifies variability in the BMG hardness and atomic-scale structure when comparing the top, middle, and bottom locations of a LPBF build with dimensions $10 \times 10 \times 18 \mathrm{~mm}^{3}$. It is important to note that these differences could not be attributed to differences in chemical composition, crystallinity,
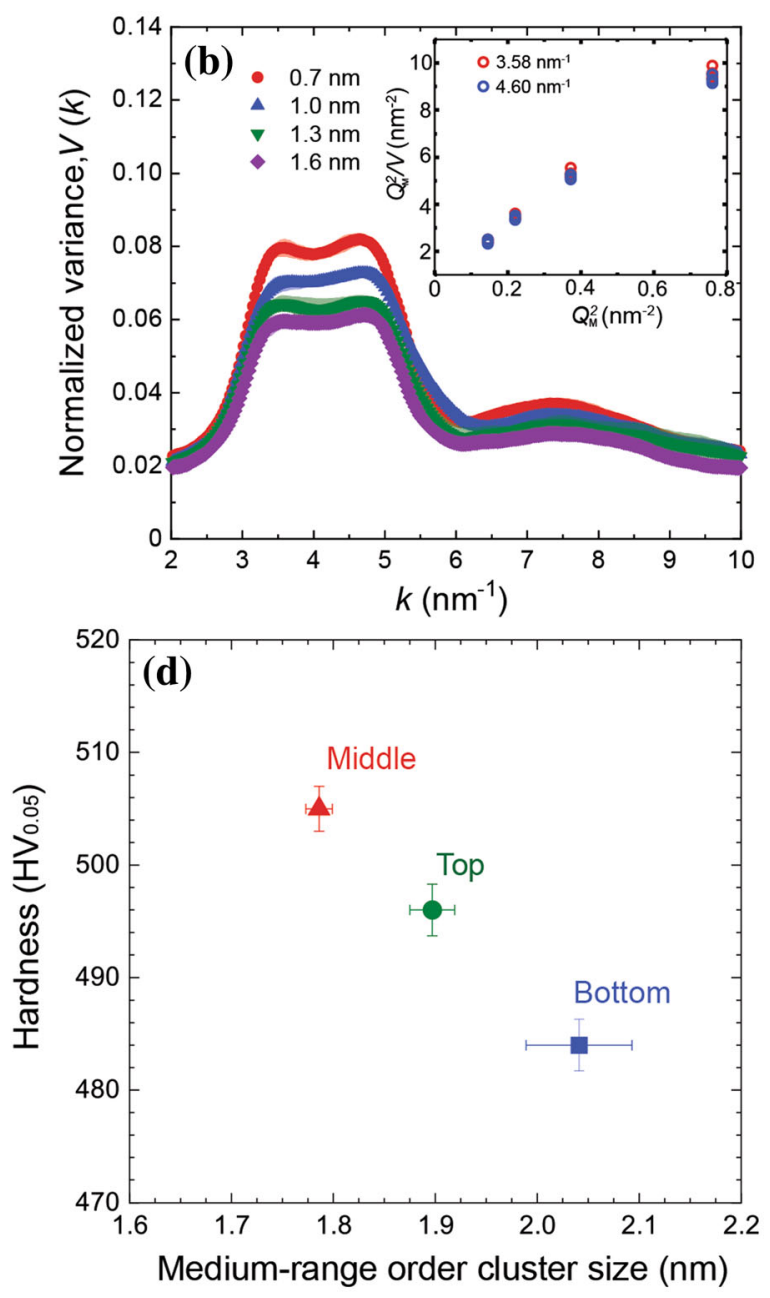

(b) was from the top of the build. Medium-range order (MRO) cluster size from the top, middle, and bottom of the LPBF AMZ4 sample using (c) electropolished TEM samples of unknown hardness and (d) site-specific TEM samples of known hardness produced via FIB lift-out. The error bars represent standard deviation for the hardness and standard error for the MRO cluster size.

or porosity at the micro- or nanoscale. Additionally, the thin specimens cut for the presented analyses are also free of residual stresses, evidenced though azimuthal integration of $I(Q)$ over $30^{\circ}$ increments on comparably thin sXRD foils published in Ref [18]. Uniform density/porosity along the build [50] and a lack of apparent crystallisation [14] has also been reported by other studies of AMZ4 produced at the same vendor using the same LPBF processing conditions.

In this study it was found that the bottom of the build was softer with a wider microhardness 
distribution that was shifted to lower values. Furthermore, the DSC curves and DB-PAS results in Figs. 3 and 4 indicated that the bottom of the build had a larger relaxation enthalpy, lower crystallisation temperature, and increased free volume. These collective results suggest that a softer, higher energy, and more rejuvenated glassy structure is present at the bottom of the build while the hardest, lowest energy, and most relaxed glassy structure occurs in the middle of the build.

To further understand the structural differences between the build locations, both sXRD and TEM were used. While our previous work found large differences in the sXRD-based structure function and PDF peak positions when comparing as-cast to LPBFprocessed AMZ4 [11] and subtle differences could be observed in the structure function across melt pools [18], differences between build locations were not detectable in the present work in both the sXRD and TEM diffraction data. However, structural differences could be observed in the MRO as revealed by NBED experiments in the TEM.

Our initial NBED experiments using electropolished TEM samples revealed the bottom sample to have larger MRO cluster size than the top and middle samples (Fig. 8a); however, those experiments examined random locations in a microstructure of variable hardness, as observed in Fig. 2. Our recent results have shown of the MRO cluster size increases linearly with decreasing local hardness, and that correlation should be independent of the processing history of the specimen [29]. This motivated a second set of NBED experiments where a FIB was used to extract TEM lamella of known hardness from the hardness maps (Fig. 2) of each region. To match the rank order of the average hardness, the softest sample was taken from the bottom and the hardest taken from the middle region. The exact hardness values chosen relative to the overall hardness distribution for each case can be seen in Fig. $2 d-f$. Figure $8 b$ shows a linear relationship between the local hardness and MRO cluster size that is in good agreement with our previous study [29]. Moreover, a larger nanoscale MRO cluster size can be considered the structural signature of the softer, more rejuvenated state found at the bottom of the build, and to a lesser extent at the top of the build.

In the LPBF process, the base-plate at the bottom of the build acts as a heat sink, helping to dissipate the applied heat by the laser energy. Thus, the bottom of the build is expected to have a higher cooling rate relative to middle locations in the build where a steady state was reached, as has been revealed by simulations of the LPBF process [27, 51, 52]. Higher cooling rates will naturally lead to a more rejuvenated glassy state which is associated with lower hardness; indeed, the relaxation enthalpy for LPBF produced AMZ4 is much higher than for its cast counterpart [12]. Both the hardness maps and the DSC results confirm this with the latter showing larger relaxation enthalpy in the bottom region. As the build progresses into the middle region, more heat must be dissipated in the part leading the slower cooling rates and cyclic reheating [27, 51]. For BMGs, this will lead to a more relaxed structural state forming in the middle of the build, and if the energy density is high enough, large amounts of crystallisation will occur in the HAZs [6]. As a result, a harder and more relaxed structure is found in the middle region and that structure should persist if a steadystate flow of heat input and dissipation is achieved. Finally, simulations have shown a slight uptick in cooling rate at the top section of LPBF builds along with a reduced amount of heat input and fewer thermal cycles relative to the steady state [52], which should result in a slightly less relaxed structure, as is observed in Figs. 2 and 3.

It is important to note that while some structural relaxation of lower layers may occur due to cyclic heat input, there does not appear to be a large amount of relaxation occurring since the relaxation enthalpy everywhere in the LPBF build remains much higher than for cast material [12]. Thus, it is thought that the cooling rate is the dominant factor controlling the structural state, and that the temperature of the solidified material must quickly fall to a low enough fraction of $T_{\mathrm{g}}$ such that continuous relaxation does not occur during the build. This is identical to reports of microstructure heterogeneity along the build height in crystalline metals, where finer scale grains, second phase cell structures, and/ or martensitic structures are observed with higher hardness at the bottom of the build due to the higher cooling rate, yet the subsequent heat input during the build is insufficient to coarsen the bottom region microstructure relative to the higher locations [53-57].

Overall, the variability in the structure and hardness seen with build height for the Zr-based BMG in this study can be explained by the variability of heat 
dissipated in the sample. To achieve more uniform microstructure and mechanical properties throughout the build, further investigations will be required, but one strategy might be to adjust the energy density at the beginning and end of the build within the available processing window to ensure a more uniform heat dissipation, while avoiding crystallisation and printing defects. In that regard, simulation studies will be an invaluable tool to guide energy density selection.

\section{Conclusions}

In this work, variations in structure and properties of a LPBF-processed BMG along its build height were elucidated using a range of advanced material characterisation techniques. These measurements revealed the structure and property differences that occur at the top, middle, and bottom regions of an LPBF-processed $\mathrm{Zr}_{59.3} \mathrm{Cu}_{28.8} \mathrm{Nb}_{1.5} \mathrm{Al}_{10.4}$ bulk metallic glass sample, where the following conclusions may be drawn.

1. The bottom region of the build was found to have a softer, higher energy, more rejuvenated glassy state than the middle region of the build, with the top region being intermediate in structure and properties.

2. Differences in hardness at different locations could not be attributed to variability in chemical composition, porosity, or crystallinity between the regions.

3. The structural signature of the softer bottom region was found to be a larger medium range order cluster size, as revealed by nanobeam electron diffraction experiments in the TEM, together with increased free volume evidenced by DSC and DB-PAS measurements.

4. Variability in the structure and properties with build height were attributed to differences in the heat dissipation at various locations in the sample. The bottom of the build experienced heat dissipation into the build plate, which gave faster cooling rates and less reheating compared to the steady-state middle of the build. Finally, at the top of the build less reheating occurs, leading to a less relaxed state compared to the middle region.

\section{Acknowledgements}

We acknowledge DESY (Hamburg, Germany), a member of the Helmholtz Association HGF, for the provision of experimental facilities. Parts of this research were carried out at PETRA III at the P07 beamline, and we thank Dr. Ann-Christin Dippel and Dr. Olof Gutowski for their support. This work is based upon experiments performed at the NEPOMUC instrument operated by FRM II at the Heinz Maier-Leibnitz Zentrum (MLZ), Garching, Germany. We also acknowledge the Deutscher Akademischer Austauschdienst (DAAD) through the AustraliaGermany Joint Research Co-operation Scheme (UADAAD, Nos. 57386795 and 57447466) and the Australian Research Council Discovery Grant No. DP180101393 for funding this work. The authors acknowledge the use of the facilities and the scientific and technical assistance of Sydney Microscopy \& Microanalysis (SMM), a core research facility of the University of Sydney and a node of Microscopy Australia-particularly Mr. Jacob Byrnes, Drs. Magnus Garbrecht, Vijay Bhatia, and Hongwei Liu.

\section{Funding}

Open Access funding enabled and organized by CAUL and its Member Institutions. Deutscher Akademischer Austauschdienst, 57386795, Zach Evenson, 57447466, Fan Yang, Australian Research Council, DP180101393, Jamie J Kruzic.

\section{Declarations}

Conflict of interest The authors declare that there is no conflict of interest associated with this research.

Supplementary Information: The online version contains supplementary material available at http s:/ /doi.org/10.1007/s10853-022-06991-6.

Open Access This article is licensed under a Creative Commons Attribution 4.0 International License, which permits use, sharing, adaptation, distribution and reproduction in any medium or format, as long as you give appropriate credit to the original author(s) and the source, provide a link to the Creative Commons licence, and indicate if changes were made. The images or other third party material in this article are included in the article's Creative Commons 
licence, unless indicated otherwise in a credit line to the material. If material is not included in the article's Creative Commons licence and your intended use is not permitted by statutory regulation or exceeds the permitted use, you will need to obtain permission directly from the copyright holder. To view a copy of this licence, visit http://creativecommons.org/licen ses/by $/ 4.0 /$.

Supplementary Information: The online version contains supplementary material available at http s://doi.org/10.1007/s10853-022-06991-6.

\section{References}

[1] Hufnagel TC, Schuh CA, Falk ML (2016) Deformation of metallic glasses: Recent developments in theory, simulations, and experiments. Acta Mater 109:375-393. https://doi.org/ 10.1016/j.actamat.2016.01.049

[2] Kruzic JJ (2016) Bulk Metallic glasses as structural materials: a review. Adv Eng Mater 18(8):1308-1331. https://doi. org/10.1002/adem.201600066

[3] Schuh CA, Hufnagel TC, Ramamurty U (2007) Overview no. 144 - mechanical behavior of amorphous alloys. Acta Mater 55 (12):4067-4109. https://doi.org/10.1016/j.actamat. 2007.01.052

[4] Suryanarayana C, Inoue A (2017) Bulk metallic glasses. CRC Press

[5] Pauly S, Löber L, Petters R, Stoica M, Scudino S, Kühn U, Eckert J (2013) Processing metallic glasses by selective laser melting. Mater Today 16(1):37-41. https://doi.org/10.1016/j. mattod.2013.01.018

[6] Li XP, Roberts MP, O'Keeffe S, Sercombe TB (2016) Selective laser melting of Zr-based bulk metallic glasses: processing, microstructure and mechanical properties. Mater Design 112:217-226. https://doi.org/10.1016/j.matdes.2016. 09.071

[7] Mahbooba Z, Thorsson L, Unosson M, Skoglund P, West H, Horn T, Rock C, Vogli E, Harrysson O (2018) Additive manufacturing of an iron-based bulk metallic glass larger than the critical casting thickness. Appl Mater Today 11:264-269. https://doi.org/10.1016/j.apmt.2018.02.011

[8] Li XP (2018) Additive manufacturing of advanced multicomponent alloys: bulk metallic glasses and high entropy alloys. Adv Eng Mater 20(5):1700874. https://doi.org/10.10 02/adem.201700874

[9] Wang L, Wang H, Liu Y, Fu Z, Peng T, Shen J, Zhou S, Yan M, Wang G, Dai Y (2019) Selective laser melting helps fabricate record-large bulk metallic glass: experiments, simulation and demonstrative part. J Alloys Compd 808:151731. https://doi.org/10.1016/j.jallcom.2019.151731

[10] Deng L, Wang S, Wang P, Kühn U, Pauly S (2018) Selective laser melting of a Ti-based bulk metallic glass. Mater Lett 212:346-349. https://doi.org/10.1016/j.matlet.2017.10.130

[11] Best JP, Ostergaard HE, Li B, Stolpe M, Yang F, Nomoto K, Hasib MT, Muránsky O, Busch R, Li X, Kruzic JJ (2020) Fracture and fatigue behaviour of a laser additive manufactured Zr-based bulk metallic glass. Addit Manuf 36:101416. https://doi.org/10.1016/j.addma.2020.101416

[12] Best JP, Ast J, Li B, Stolpe M, Busch R, Yang F, Li X, Michler J, Kruzic JJ (2020) Relating fracture toughness to micro-pillar compression response for a laser powder bed additive manufactured bulk metallic glass. Mater Sci Eng A 770:138535. https://doi.org/10.1016/j.msea.2019.138535

[13] Sohrabi N, Jhabvala J, Kurtuldu G, Stoica M, Parrilli A, Berns S, Polatidis E, Van Petegem S, Hugon S, Neels A, Löffler JF, Logé RE (2021) Characterization, mechanical properties and dimensional accuracy of a Zr-based bulk metallic glass manufactured via laser powder-bed fusion. Mater Des 199:109400. https://doi.org/10.1016/j.matdes.20 20.109400

[14] Bordeenithikasem P, Stolpe M, Elsen A, Hofmann DC (2018) Glass forming ability, flexural strength, and wear properties of additively manufactured Zr-based bulk metallic glasses produced through laser powder bed fusion. Addit Manuf 21:312-317. https://doi.org/10.1016/j.addma.2018.0 3.023

[15] Deng L, Gebert A, Zhang L, Chen HY, Gu DD, Kühn U, Zimmermann M, Kosiba K, Pauly S (2020) Mechanical performance and corrosion behaviour of $\mathrm{Zr}$-based bulk metallic glass produced by selective laser melting. Mater Design 189:108532. https://doi.org/10.1016/j.matdes.2020. 108532

[16] Wegner J, Frey M, Piechotta M, Neuber N, Adam B, Platt S, Ruschel L, Schnell N, Riegler SS, Jiang H-R, Witt G, Busch $R$, Kleszczynski S (2021) Influence of powder characteristics on the structural and the mechanical properties of additively manufactured Zr-based bulk metallic glass. Mater Des 209:109976. https://doi.org/10.1016/j.matdes.2021.109976

[17] Ritchie RO (2011) The conflicts between strength and toughness. Nat Mater 10(11):817-822. https://doi.org/10.10 38/Nmat3115

[18] Best JP, Evenson Z, Yang F, Dippel A-C, Stolpe M, Gutowski O, Hasib MT, Li X, Kruzic JJ (2019) Structural periodicity in laser additive manufactured Zr-based bulk metallic glass. Appl Phys Lett 115(3):031902. https://doi. org/10.1063/1.5100050

[19] Li BS, Shahabi HS, Scudino S, Eckert J, Kruzic JJ (2015) Designed heterogeneities improve the fracture reliability of a 
Zr-based bulk metallic glass. Mater Sci Eng A-Struct Mater Prop Microstruct Process 646:242-248. https://doi.org/10.1 016/j.msea.2015.08.055

[20] Li BS, Xie S, Kruzic JJ (2019) Toughness enhancement and heterogeneous softening of a cryogenically cycled $\mathrm{Zr}-\mathrm{Cu}-$ $\mathrm{Ni}-\mathrm{Al}-\mathrm{Nb}$ bulk metallic glass. Acta Mater 176:278-288. h ttps://doi.org/10.1016/j.actamat.2019.07.012

[21] Xie SH, Kruzic JJ (2017) Cold rolling improves the fracture toughness of a Zr-based bulk metallic glass. J Alloys Compd 694:1109-1120

[22] Li BS, Scudino S, Gludovatz B, Kruzic JJ (2020) Role of pre-existing shear band morphology in controlling the fracture behavior of a $\mathrm{Zr}-\mathrm{Ti}-\mathrm{Cu}-\mathrm{Ni}-\mathrm{Al}$ bulk metallic glass. Mater Sci Eng A 786:139396

[23] Sun H, Flores KM (2008) Laser deposition of a Cu-based metallic glass powder on a Zr-based glass substrate. J Mater Res 23(10):2692-2703. https://doi.org/10.1557/JMR.2008. 0329

[24] Ouyang D, Xing W, Li N, Li Y, Liu L (2018) Structural evolutions in 3D-printed Fe-based metallic glass fabricated by selective laser melting. Addit Manuf 23:246-252. http s://doi.org/10.1016/j.addma.2018.08.020

[25] Ouyang D, Li N, Liu L (2018) Structural heterogeneity in 3D printed Zr-based bulk metallic glass by selective laser melting. J Alloy Compd 740:603-609. https://doi.org/10.10 16/j.jallcom.2018.01.037

[26] Chen W, Yang Q, Huang S, Huang S, Kruzic JJ, Li X (2021) Laser power modulated microstructure evolution, phase transformation and mechanical properties in NiTi fabricated by laser powder bed fusion. J Alloy Compd 861:157959. h ttps://doi.org/10.1016/j.jallcom.2020.157959

[27] DebRoy T, Wei HL, Zuback JS, Mukherjee T, Elmer JW, Milewski JO, Beese AM, Wilson-Heid A, De A, Zhang W (2018) Additive manufacturing of metallic componentsprocess, structure and properties. Prog Mater Sci 92:112-224. https://doi.org/10.1016/j.pmatsci.2017.10.001

[28] Kok Y, Tan XP, Wang P, Nai MLS, Loh NH, Liu E, Tor SB (2018) Anisotropy and heterogeneity of microstructure and mechanical properties in metal additive manufacturing: a critical review. Mater Des 139:565-586. https://doi.org/10. 1016/j.matdes.2017.11.021

[29] Nomoto K, Ceguerra AV, Gammer C, Li B, Bilal H, Hohenwarter A, Gludovatz B, Eckert J, Ringer SP, Kruzic JJ (2021) Medium-range order dictates local hardness in bulk metallic glasses. Mater Today 44:48-57. https://doi.org/10. 1016/j.mattod.2020.10.032

[30] Gigl T, Beddrich L, Dickmann M, Rienäcker B, Thalmayr M, Vohburger S, Hugenschmidt C (2017) Defect imaging and detection of precipitates using a new scanning positron microbeam. New J Phys 19(12):123007. https://doi.org/10. 1088/1367-2630/aa915b

[31] Hugenschmidt C, Piochacz C, Reiner M, Schreckenbach K (2012) The NEPOMUC upgrade and advanced positron beam experiments. New J Phys 14(5):055027. https://doi. org/10.1088/1367-2630/14/5/055027

[32] Kieffer J, Karkoulis D (2013) PyFAI, a versatile library for azimuthal regrouping. J Phys: Conf Ser 425(20):202012. h ttps://doi.org/10.1088/1742-6596/425/20/202012

[33] Juhas P, Davis T, Farrow CL, Billinge SJL (2013) PDFgetX3: a rapid and highly automatable program for processing powder diffraction data into total scattering pair distribution functions. J Appl Crystallogr 46(2):560-566. h ttps://doi.org/10.1107/S0021889813005190

[34] Warren BE (1937) X-ray determination of the structure of liquids and glass. J Appl Phys 8(10):645-654. https://doi. org/10.1063/1.1710241

[35] Cockayne DJH, McKenzie DR (1988) Electron diffraction analysis of polycrystalline and amorphous thin films. Acta Crystallogr A 44(6):870-878. https://doi.org/10.1107/ S0108767388004957

[36] Mattern N, Kühn U, Hermann H, Ehrenberg H, Neuefeind J, Eckert J (2002) Short-range order of Zr62-xTixA110Cu20Ni8 bulk metallic glasses. Acta Mater 50(2):305-314. https://doi.org/10.1016/S1359-6454(01)003 41-X

[37] Hwang J, Voyles PM (2011) Variable resolution fluctuation electron microscopy on $\mathrm{Cu}-\mathrm{Zr}$ metallic glass using a wide range of coherent STEM probe size. Microsc Microanal 17(1):67-74. https://doi.org/10.1017/S1431927610094109

[38] Treacy MMJ, Gibson JM, Fan L, Paterson DJ, McNulty I (2005) Fluctuation microscopy: a probe of medium range order. Rep Prog Phys 68(12):2899-2944. https://doi.org/10. 1088/0034-4885/68/12/r06

[39] Bogle SN, Nittala LN, Twesten RD, Voyles PM, Abelson JR (2010) Size analysis of nanoscale order in amorphous materials by variable-resolution fluctuation electron microscopy. Ultramicroscopy 110(10):1273-1278. https://doi.org/ 10.1016/j.ultramic.2010.05.001

[40] Gibson JM, Treacy MMJ, Voyles PM (2000) Atom pair persistence in disordered materials from fluctuation microscopy. Ultramicroscopy 83(3):169-178. https://doi.org/10.1 016/S0304-3991(00)00013-9

[41] Voyles PM, Muller DA (2002) Fluctuation microscopy in the STEM. Ultramicroscopy 93(2):147-159. https://doi.org/10. 1016/S0304-3991(02)00155-9

[42] Daulton TL, Bondi KS, Kelton KF (2010) Nanobeam diffraction fluctuation electron microscopy technique for structural characterization of disordered materials - application to Al88-xY7Fe5Tix metallic glasses. Ultramicroscopy 
110(10):1279-1289. https://doi.org/10.1016/j.ultramic.2010. 05.010

[43] Gammer C, Mangler C, Rentenberger C, Karnthaler HP (2010) Quantitative local profile analysis of nanomaterials by electron diffraction. Scripta Mater 63(3):312-315. http s://doi.org/10.1016/j.scriptamat.2010.04.019

[44] Gammer C, Escher B, Ebner C, Minor AM, Karnthaler HP, Eckert J, Pauly S, Rentenberger C (2017) Influence of the Ag concentration on the medium-range order in a $\mathrm{CuZrAlAg}$ bulk metallic glass. Sci Rep-Uk 7(1):44903. https://doi.org/ 10.1038/srep44903

[45] Stolpe M, Kruzic JJ, Busch R (2014) Evolution of shear bands, free volume and hardness during cold rolling of a $\mathrm{Zr}$ based bulk metallic glass. Acta Mater 64:231-240. https://d oi.org/10.1016/j.actamat.2013.10.035

[46] Ramachandramoorthy R, Yang F, Casari D, Stolpe M, Jain M, Schwiedrzik J, Michler J, Kruzic JJ, Best JP (2021) High strain rate in situ micropillar compression of a Zr-based metallic glass. J Mater Res 36(11):2325-2336. https://doi. org/10.1557/s43578-021-00187-5

[47] Sun YJ, Qu DD, Huang YJ, Liss KD, Wei XS, Xing DW, Shen J (2009) $\mathrm{Zr}-\mathrm{Cu}-\mathrm{Ni}-\mathrm{Al}$ bulk metallic glasses with superhigh glass-forming ability. Acta Mater 57(4):1290-1299. https://doi.org/10.1016/j.actamat.2008.11. 007

[48] Liu S, Wang L, Ge J, Wu Z, Ke Y, Li Q, Sun B, Feng T, Wu Y, Wang JT, Hahn H, Ren Y, Almer JD, Wang X-1, Lan S (2020) Deformation-enhanced hierarchical multiscale structure heterogeneity in a Pd-Si bulk metallic glass. Acta Mater 200:42-55. https://doi.org/10.1016/j.actamat.2020.08.077

[49] Stoica M, Das J, Bednarcik J, Franz H, Mattern N, Wang WH, Eckert J (2008) Strain distribution in Zr64.13Cu15.75Ni10.12A110 bulk metallic glass investigated by in situ tensile tests under synchrotron radiation. J Appl Phys 104(1):013522. https://doi.org/10.1063/1. 2952034

[50] Shi J, Ma S, Wei S, Best JP, Stolpe M, Beckmann A, Mostafavi S, Korte-Kerzel S, Markert B (2020) 3D pore structure characterization and hardness in a powder bed fusion-processed fully amorphous Zr-based bulk metallic glass. Mater Charact 162:110178. https://doi.org/10.1016/j. matchar.2020.110178

[51] Mukherjee T, Zhang W, DebRoy T (2017) An improved prediction of residual stresses and distortion in additive manufacturing. Comput Mater Sci 126:360-372. https://doi. org/10.1016/j.commatsci.2016.10.003

[52] Wang XQ, Chou K (2019) Microstructure simulations of Inconel 718 during selective laser melting using a phase field model. Int J Adv Manuf Technol 100(9-12):2147-2162. h ttps://doi.org/10.1007/s00170-018-2814-Z

[53] Wang XQ, Keya T, Chou K (2016) Build height effect on the inconel 718 parts fabricated by selective laser melting. Proc Manufact 5:1006-1017. https://doi.org/10.1016/j.promfg.20 16.08.089

[54] Yao Y, Wang KW, Wang XQ, Li L, Cai WJ, Kelly S, Esparragoza N, Rosser M, Yan F (2020) Microstructural heterogeneity and mechanical anisotropy of $18 \mathrm{Ni}-330$ maraging steel fabricated by selective laser melting: The effect of build orientation and height. J Mater Res 35(15):2065-2076. https://doi.org/10.1557/jmr.2020.126

[55] Zhang B, Wang P, Chew Y, Wen Y, Zhang M, Wang P, Bi G, Wei J (2020) Mechanical properties and microstructure evolution of selective laser melting Inconel 718 along building direction and sectional dimension. Mater Sci Eng A 794:139941. https://doi.org/10.1016/j.msea.2020.139941

[56] Mohr G, Altenburg SJ, Hilgenberg K (2020) Effects of inter layer time and build height on resulting properties of $316 \mathrm{~L}$ stainless steel processed by laser powder bed fusion. Addit Manuf 32:101080. https://doi.org/10.1016/j.addma.2020. 101080

[57] Palanivel S, Dutt AK, Faierson EJ, Mishra RS (2016) Spatially dependent properties in a laser additive manufactured Ti-6Al-4V component. Mater Sci Eng, A 654:39-52. http s://doi.org/10.1016/j.msea.2015.12.021

Publisher's Note Springer Nature remains neutral with regard to jurisdictional claims in published maps and institutional affiliations. 